\title{
High-resolution 3D dust radiative transfer in galaxies with DART-Ray
}

\author{
Giovanni Natale ${ }^{1,2}$, Cristina C. Popescu ${ }^{1,2}$, Richard. J. Tuffs ${ }^{2}$, \\ Victor P. Debattista ${ }^{1}$ and Meiert W. Grootes ${ }^{2}$ \\ ${ }^{1}$ Jeremiah Horrocks Institute, University of Central Lancashire, Preston, PR1 2HE, UK \\ ${ }^{2}$ Max Planck Institute für Kernphysik, Saupfercheckweg 1, D-69117 Heidelberg, Germany
}

\begin{abstract}
DART-Ray is a 3D ray-tracing dust radiative transfer (RT) code that can be used to derive stellar and dust emission maps of galaxy models and simulations with arbitrary geometries. In addition to the previously published RT algorithm, we have now included in DART-Ray the possibility of calculating the stocastically heated dust emission from each volume element within a galaxy. To show the capabilities of the code, we performed a high-resolution (26 pc) $\mathrm{RT}$ calculation for a galaxy N-body+SPH simulation. The simulated galaxy we considered is characterized by a nuclear disc and a flocculent spiral structure. We analysed the derived galaxy maps for the global and local effects of dust on the galaxy attenuation as well as the contribution of scattered radiation to the predicted observed emission. In addition, by performing an additional RT calculation including only the stellar volume emissivity due to young stellar populations (SPs), we derived the contribution to the total dust emission powered by young and old SPs. Full details of this work will be presented in a forthcoming publication.
\end{abstract}
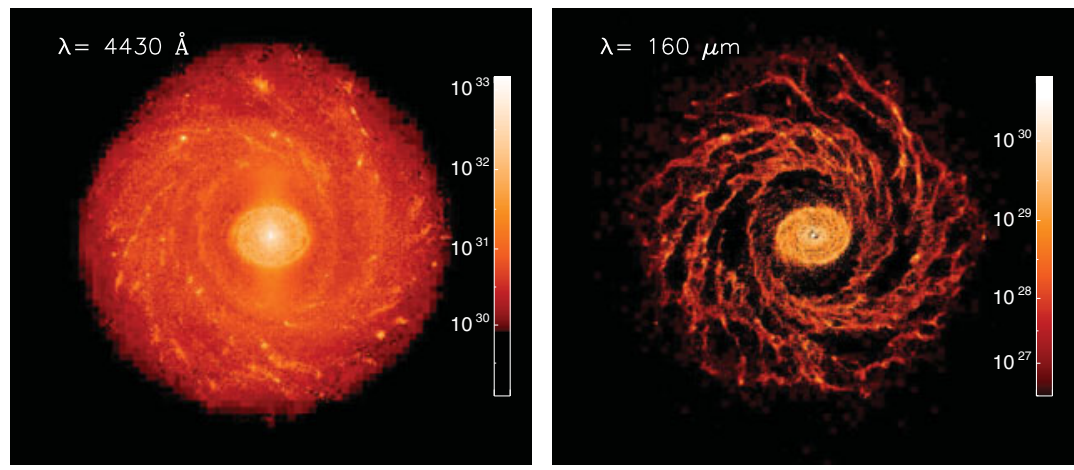

Figure 1. Predicted face-on maps for a galaxy Nbody+SPH simulation. The left panel shows the stellar emission map at $4430 \AA$ (within the B-band) and the right panel the dust emission map at $160 \mu \mathrm{m}$. The units of the values aside of the colour bar are $\mathrm{erg} /\left(\mathrm{s} \AA \mathrm{sr} \mathrm{pc}{ }^{2}\right)$.

\section{References}

Cole, D. et al. 2014, submitted

Natale, G., Popescu, C. C., Tuffs, R. J., \& Semionov, D. 2014, MNRAS, 438, 3137

Natale, G. et al. 2014, in prep.

Popescu, C. C., Tuffs, R. J., Dopita, M. A., Fischera, J., et al. 2011, A\&\&A, 527, 109 